\title{
Rayleigh waves of arbitrary profile in anisotropic media
}

\author{
D. A. Prikazchikov
}

Dept. of Computational Mathematics and Mathematical Physics,

The Bauman Moscow State Technical University, Moscow, Russia

\begin{abstract}
The paper deals with surface wave propagation in an orthorhombic elastic half-plane. The general profile of the wave is considered, incorporating the anisotropy effects within the known representation in terms of a single plane harmonic function.
\end{abstract}

Keywords: surface waves, arbitrary profile, single harmonic function.

\footnotetext{
${ }^{2}$ Currently at Department of Mathematics, Keele University, Keele, Staffordshire, UK, ST5 5BG
} 


\section{Introduction and governing equations}

Surface wave propagation in anisotropic media is well-studied, see e.g. Destrade (2001) and references therein. However, the majority of the contributions deal with harmonic travelling waves of the form $e^{i k\left(x_{1}-v t\right)}$. Chadwick (1976), inspired by the work of Friedlander (1948), has presented a more general solution for the Rayleigh wave field in terms of a single harmonic function in case of a linearly isotropic elastic half-plane. This approach was later extended to interfacial waves and surface waves with general lateral dependence, see Kiselev and Parker (2010), and Kiselev and Rogerson (2009). Achenbach (1998) has shown for time-harmonic disturbances that in case of a transversely isotropic half-space the solution for surface waves at any depth is governed by a membrane equation.

The aim of this note is to investigate further surface waves of arbitrary profile in case of anisotropic media. In particular, the problem is considered for an orthorhombic elastic half-plane $\left(-\infty<x_{1}<\infty, 0 \leq x_{2}<\infty\right)$. Following Chadwick (1976), a general representation for the free surface wave field is obtained through an arbitrary plane harmonic function.

The equations of motion of linear elasticity are given by

$$
\sigma_{j m, m}=\rho \ddot{u}_{j},
$$

where $\rho$ is volume density, $u_{j}$ and $\sigma_{j m}(j, m=1,2)$ are the displacement and traction components, respectively, a dot conventionally denotes differentiation with respect to time $t$, a comma indicates differentiation along the corresponding spatial variable, and summation over repeated indices is assumed.

The constitutive relations for the orthorhombic elastic solid are written explicitly as

$$
\sigma_{11}=c_{11} u_{1,1}+c_{12} u_{2,2}, \quad \sigma_{22}=c_{12} u_{1,1}+c_{22} u_{2,2}, \quad \sigma_{12}=c_{66}\left(u_{1,2}+u_{2,1}\right),
$$

where $c_{11}, c_{12}, c_{22}$ and $c_{66}$ are stiffness components, satisfying the conditions

$$
c_{11}>0, \quad c_{11} c_{22}-c_{12}^{2}>0, \quad c_{66}>0,
$$

required for the positive definiteness of the strain-energy density, see Ting (1996).

Substituting (2) into (1), one arrives at

$$
\begin{aligned}
& c_{11} u_{1,11}+c_{66} u_{1,22}+\beta u_{2,12}=\rho \ddot{u}_{1}, \\
& \beta u_{1,12}+c_{66} u_{2,11}+c_{22} u_{2,22}=\rho \ddot{u}_{2},
\end{aligned}
$$

with $\beta=c_{12}+c_{66}$.

The free boundary conditions are imposed at the surface $x_{2}=0$ of the half-plane

$$
\sigma_{j 2}=0, \quad(j=1,2) .
$$

\section{Surface waves of arbitrary profile}

Similarly to Chadwick (1976), we start from the ansatz in the form of a travelling wave, namely $(j=1,2)$

$$
u_{j}=U_{j}\left(x_{1}-v t, x_{2}\right) .
$$


Substitution of the latter into the equations of motion (4) gives

$$
\begin{aligned}
& \left(c_{11}-\bar{v}^{2}\right) U_{1,11}+c_{66} U_{1,22}+\beta U_{2,12}=0 \\
& \beta U_{1,12}+\left(c_{66}-\bar{v}^{2}\right) U_{2,11}+c_{22} U_{2,22}=0
\end{aligned}
$$

with $\bar{v}^{2}=\rho v^{2}$.

Equations (7) may be transformed to a fourth order PDE in respect of one of the sought for displacements. For example, in terms of $U_{1}$ we obtain

$$
U_{1,1111}+a_{1} U_{1,1122}+a_{2} U_{1,2222}=0
$$

where

$$
a_{1}=\frac{c_{66}^{2}+c_{11} c_{22}-\beta^{2}-\left(c_{22}+c_{66}\right) \bar{v}^{2}}{c_{22} c_{66}}, \quad a_{2}=\frac{\left(c_{11}-\bar{v}^{2}\right)\left(c_{66}-\bar{v}^{2}\right)}{c_{22} c_{66}} .
$$

It may be shown that the obtained fourth order equation (8) is elliptic, with the coefficients $a_{1}$ and $a_{2}$ coinciding with that of the traditional characteristic equation for attenuation orders, see e.g. Royer and Dieulesaint (1996). It may therefore be presented in operator form as

$$
\left[\partial_{2}^{2}+\lambda_{1}^{2} \partial_{1}^{2}\right]\left[\partial_{2}^{2}+\lambda_{2}^{2} \partial_{1}^{2}\right] U_{1}=0
$$

where

$$
\lambda_{1}^{2}+\lambda_{2}^{2}=a_{1}, \quad \lambda_{1}^{2} \lambda_{2}^{2}=a_{2}
$$

Hence, the solution of (10) may be expressed as

$$
U_{1}=\sum_{j=1}^{2} \phi_{j}\left(x_{1}-v t, \lambda_{j} x_{2}\right),
$$

where $\phi_{j}$ are arbitrary plane harmonic functions, decaying as $x_{2} \rightarrow \infty$. Bearing in mind the obtained solution (12) and using the Cauchy-Riemann identities for plane harmonic function in the form

$$
\frac{\partial \phi}{\partial x_{2}}=-\lambda \frac{\partial \phi^{*}}{\partial x_{1}}, \quad \frac{\partial \phi}{\partial x_{1}}=\frac{1}{\lambda} \frac{\partial \phi^{*}}{\partial x_{2}}, \quad \phi=\phi\left(x_{1}, \lambda x_{2}\right),
$$

it is possible to express another displacement from $(7)_{1}$ as

$$
U_{2}=\sum_{j=1}^{2} f\left(\lambda_{j}, \bar{v}^{2}\right) \phi_{j}^{*}\left(x_{1}-v t, \lambda_{j} x_{2}\right),
$$

with the asterisk denoting the harmonic conjugate and

$$
f\left(\lambda, \bar{v}^{2}\right)=\frac{c_{66} \lambda^{2}-c_{11}+\bar{v}^{2}}{\beta \lambda} .
$$


The solutions (12), (14) may now be substituted into the free surface boundary conditions (5). Using the Cauchy-Riemann identities, we arrive at

$$
\begin{aligned}
& \sum_{j=1}^{2}\left(f\left(\lambda_{j}, \bar{v}^{2}\right)-\lambda_{j}\right) \phi_{j, 1}\left(x_{1}-v t, 0\right)=0 \\
& \sum_{j=1}^{2}\left(c_{12}+c_{22} \lambda_{j} f\left(\lambda_{j}, \bar{v}^{2}\right)\right) \phi_{j, 1}\left(x_{1}-v t, 0\right)=0
\end{aligned}
$$

which possesses non-trivial solutions provided the associate determinant is non-zero. After some small algebraic manipulations the latter may be rewritten as a well-known bi-cubic

$$
c_{22} c_{66} \bar{v}^{4}\left(c_{11}-\bar{v}^{2}\right)-\left(c_{66}-\bar{v}^{2}\right)\left[c_{22}\left(c_{11}-\bar{v}^{2}\right)-c_{12}^{2}\right]^{2}=0,
$$

see e.g. Royer and Dieulesaint (1996). Then, similarly to Chadwick (1976), it is possible to express the displacement components in terms of a single harmonic function. Indeed, using $(16)_{1}$ and the properties of harmonic functions, $\phi_{1}$ and $\phi_{2}$ may be related as

$$
\phi_{2}\left(\xi, \lambda_{2} x_{2}\right)=-h\left(\bar{v}_{R}^{2}\right) \phi_{1}\left(\xi, \lambda_{2} x_{2}\right)
$$

where $v=v_{R}$ is the surface wave speed being the root of (17), and

$$
\xi=x_{1}-v_{R} t, \quad \bar{v}_{R}^{2}=\rho v_{R}^{2}, \quad h\left(\bar{v}_{R}^{2}\right)=\frac{f\left(\lambda_{1}, \bar{v}_{R}^{2}\right)-\lambda_{1}}{f\left(\lambda_{2}, \bar{v}_{R}^{2}\right)-\lambda_{2}} .
$$

Therefore, in terms of $\phi_{1}$ we have

$$
\begin{aligned}
& U_{1}\left(\xi, x_{2}\right)=\phi_{1}\left(\xi, \lambda_{1} x_{2}\right)-h\left(\bar{v}_{R}^{2}\right) \phi_{1}\left(\xi, \lambda_{2} x_{2}\right), \\
& U_{2}\left(\xi, x_{2}\right)=f\left(\lambda_{1}, \bar{v}_{R}^{2}\right) \phi_{1}^{*}\left(\xi, \lambda_{1} x_{2}\right)-f\left(\lambda_{2}, \bar{v}_{R}^{2}\right) h\left(\bar{v}_{R}^{2}\right) \phi_{1}^{*}\left(\xi, \lambda_{2} x_{2}\right) .
\end{aligned}
$$

\section{Examples}

In this section we present several examples illustrating the proposed representation (19).

\subsection{Sinusoidal profile}

Let us begin with the well-known sinusoidal profile, being a particular case of the obtained representation. In this case the harmonic function $\phi_{1}$ in (12) may be specified as

$$
\phi_{1}\left(\xi, \lambda x_{2}\right)=\sin \xi e^{\lambda x_{2}}, \quad \operatorname{Re}(\lambda)<0,
$$

therefore the expressions for the displacement field (19) are given by

$$
\begin{aligned}
& U_{1}\left(\xi, x_{2}\right)=\left[e^{\lambda_{1} x_{2}}-h\left(\bar{v}_{R}^{2}\right) e^{\lambda_{2} x_{2}}\right] \sin \xi \\
& U_{2}\left(\xi, x_{2}\right)=\left[f\left(\lambda_{1}, v_{R}^{2}\right) e^{\lambda_{1} x_{2}}-f\left(\lambda_{2}, v_{R}^{2}\right) h\left(\bar{v}_{R}^{2}\right) e^{\lambda_{2} x_{2}}\right] \cos \xi
\end{aligned}
$$


where $\operatorname{Re}\left(\lambda_{j}\right)<0,(j=1,2)$ ensure the decay conditions. It follows from (11), that the attenuation coefficients $\lambda_{1}$ and $\lambda_{2}$ could be either real or complex conjugates. In the latter case $\left(\lambda_{1,2}=-a \pm i b, a, b>0\right)$ the expressions (21) may be rewritten as

$$
\tilde{U}_{1}=F_{1}\left(x_{2}\right) \sin \xi, \quad \tilde{U}_{2}=F_{2}\left(x_{2}\right) \cos \xi,
$$

where

$$
\tilde{U}_{j}=i\left(\lambda_{2}-f\left(\lambda_{2}, v_{R}^{2}\right)\right) U_{j}\left(\xi, x_{2}\right) \quad(j=1,2)
$$

and

$$
\begin{aligned}
F_{1}\left(x_{2}\right) & =\frac{2}{\beta}\left(\frac{\bar{v}_{R}^{2}-c_{11}}{a^{2}+b^{2}}+c_{12}\right) e^{-a x_{2}}\left[b \cos \left(b x_{2}\right)+a \sin \left(b x_{2}\right)\right], \\
F_{2}\left(x_{2}\right) & =\frac{2 e^{-a x_{2}}}{\beta\left(a^{2}+b^{2}\right)}\left\{\left[\left(c_{11}-\bar{v}_{R}^{2}\right)^{2}+\left(c_{11}-\bar{v}_{R}^{2}\right)\left(c_{12}-c_{66}\right)\left(a^{2}-b^{2}\right)\right.\right. \\
& \left.\left.-c_{12} c_{66}\left(a^{2}+b^{2}\right)^{2}\right] \sin \left(b x_{2}\right)+2 a b \beta\left(c_{11}-\bar{v}_{R}^{2}\right) \cos \left(b x_{2}\right)\right\} .
\end{aligned}
$$

It may be shown that the eigenfunctions $\tilde{U}_{j}$ are real.

A typical illustration of this case is presented in 1, showing the decay of the functions $F_{j}(j=1,2)$ with depth. The numerical computations within this section are performed for the $\alpha$-iodic acid $\left(\mathrm{HIO}_{3}\right)$ with the material parameters given by $c_{11}=30.1, c_{12}=16.1$, $c_{22}=58, c_{66}=15.8(\mathrm{GPa})$, and density $\rho=4640\left(\mathrm{~kg} / \mathrm{m}^{3}\right)$, see Royer and Dieulesaint (1996).
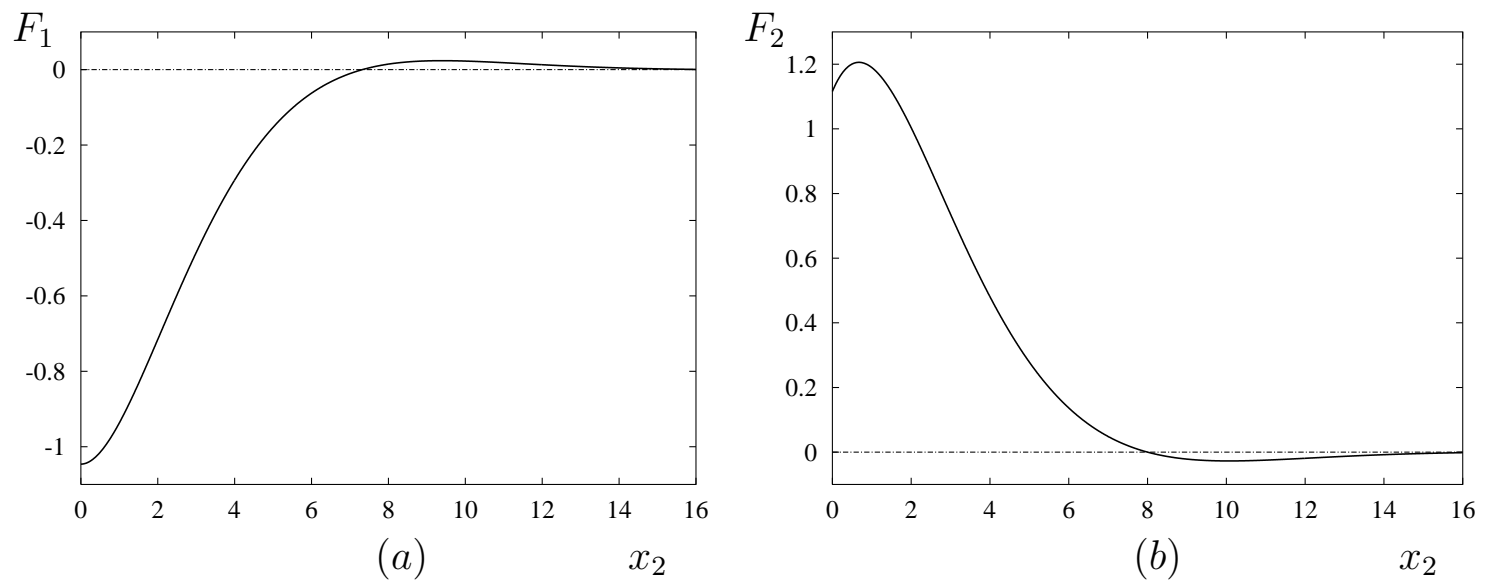

Figure 1: Decaying components for the (a) horizontal and (b) vertical displacements of sinusoidal profile.

\subsection{Localized profile}

Let us now consider the surface wave field corresponding to the decaying harmonic function in (12) specified as

$$
\phi_{1}\left(\xi, \lambda x_{2}\right)=\frac{\xi}{\xi^{2}+\left(\lambda x_{2}+\delta\right)^{2}},
$$

implying the conjugate function

$$
\phi_{1}^{*}\left(\xi, \lambda x_{2}\right)=-\frac{\lambda x_{2}+\delta}{\xi^{2}+\left(\lambda x_{2}+\delta\right)^{2}},
$$


where $\delta>0$ is a constant. The displacement field (19) is then written as

$$
\begin{aligned}
U_{1}\left(\xi, x_{2}\right) & =\frac{\xi}{\xi^{2}+\left(\lambda_{1} x_{2}+\delta\right)^{2}}-\frac{h\left(\bar{v}_{R}^{2}\right) \xi}{\xi^{2}+\left(\lambda_{2} x_{2}+\delta\right)^{2}}, \\
U_{2}\left(\xi, x_{2}\right) & =\frac{h\left(\bar{v}_{R}^{2}\right) f\left(\lambda_{2}, \bar{v}_{R}^{2}\right)\left(\lambda_{2} x_{2}+\delta\right)}{\xi^{2}+\left(\lambda_{2} x_{2}+\delta\right)^{2}}-\frac{f\left(\lambda_{1}, \bar{v}_{R}^{2}\right)\left(\lambda_{1} x_{2}+\delta\right)}{\xi^{2}+\left(\lambda_{1} x_{2}+\delta\right)^{2}} .
\end{aligned}
$$

Similarly to the previous example, it may be shown that the scaled displacements $\tilde{U}_{j}$ defined in (23), are real.

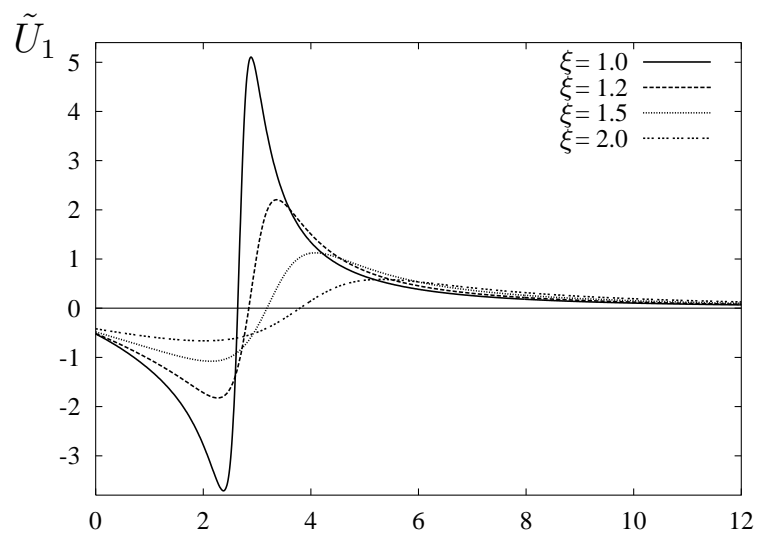

$(a)$

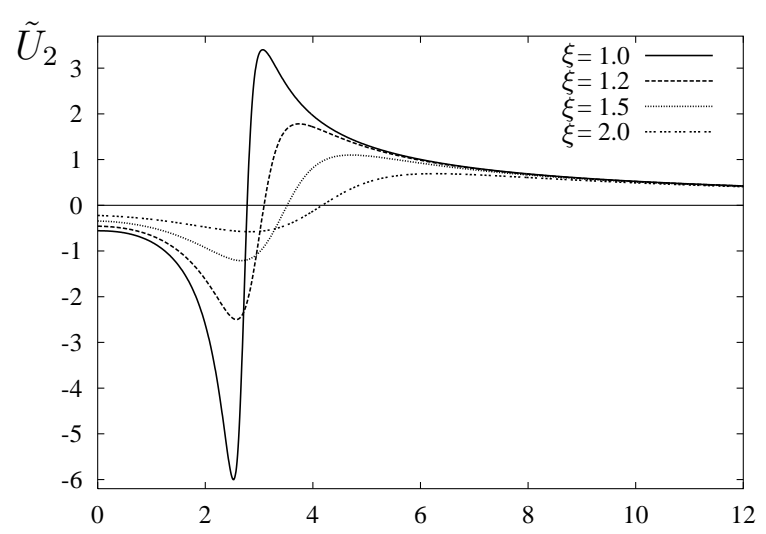

(c)

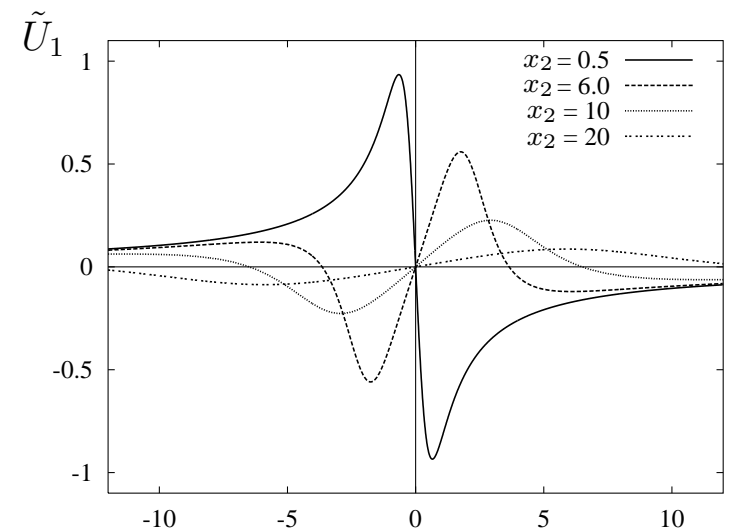

(b)

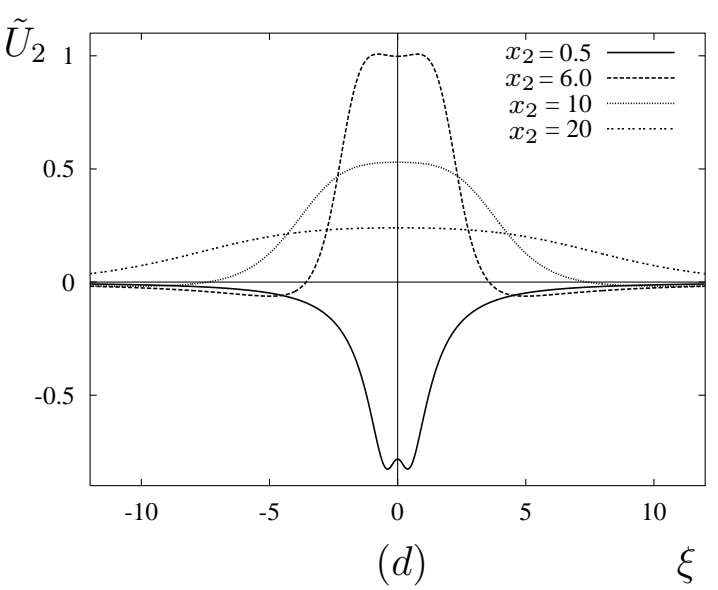

$(d)$ $\xi$

Figure 2: Localized horizontal and vertical displacements $(\delta=1)$.

Finally, we present numerical illustrations of the obtained solutions, see Figure 2. The decay of the horizontal displacement $\tilde{U}_{1}$ along the transverse variable $x_{2}$ is presented in Figure $2 \mathrm{a}$ for several values of $\xi$, while the variation along $\xi$ at several depths is shown in Figure 2b. Similar graphs for the vertical displacement $\tilde{U}_{2}$ are given in Figures $2 \mathrm{c}$ and $2 \mathrm{~d}$, respectively.

\section{Concluding remarks}

The main outcome of this note is the formulation (19) for surface wave field in terms of a single harmonic function in case of an orthorhombic elastic half-plane, which extends the previously known results for linearly isotropic media. 
As shown in Royer and Dieulesaint (1996), due to the general nature of the surface wave equation (17), the obtained results are also valid for the cases of tetragonal, hexagonal and cubic crystals. It should also be possible to generalize the presented approach for pre-stressed elastic media (Dowaikh and Ogden , 1991), 3D case, see also Destrade (2004), and interfacial waves (Parker , 2012).

A similar treatment may also be performed for the flexural edge wave in anisotropic plate, however, the travelling wave ansatz should be replaced by the beam-like assumption, for more details see Kaplunov et al. (2013) and references therein.

Another issue is concerned with the specialized hyperbolic-elliptic formulations for surface dynamics in case of the forced boundary value problems, see e.g. Kaplunov et al. (2006). The results established in the present note for free surface waves provide a path to development of the asymptotic models for surface waves in anisotropic media including the cases of coated half-space (Dai et al. , 2010), and mixed problems (Erbaş et al. , 2012). The obtained asymptotic formulations may also be applied to various moving load problems, see Kaplunov et al. (2010) and references therein.

\section{Acknowledgement}

This study was supported by The Ministry of Education and Science of Russia, project 14.A18.21.2014. Fruitful discussions with Prof J. Kaplunov and valuable comments of the anonymous referee are also gratefully acknowledged.

\section{References}

Achenbach, J.D., 1998. Explicit solutions for carrier waves supporting surface waves and plate waves. Wave Motion 28, 89-97.

Chadwick, P., 1976. Surface and interfacial waves of arbitrary form in isotropic elastic media. J. Elast. 6, 73-80.

Dai H.H., Kaplunov, J., Prikazchikov, D.A., 2010. A long-wave model for the surface elastic wave in a coated half-space. Proc. Roy. Soc. A. 466, 3097-3116.

Destrade, M., 2001. The explicit secular equation for surface acoustic waves in monoclinic elastic crystals. J. Acoust. Soc. Am. 109(4), 1398-1402.

Destrade M., 2004. Surface acoustic waves in rotating orthorhombic crystals. Proc. Roy. Soc. A. 460, 653-665.

Dowaikh M.A, Ogden R.W., 1991. On surface waves and deformations in a compressible elastic half space. SAACM 1, 27-45.

Erbaş, B., Kaplunov, J., Prikazchikov,D.A., 2012. The Rayleigh wave field in mixed problems for a half-plane. IMA J. Appl. Math. Published online (doi: 10.1093/imamat/hxs010).

Friedlander, F.G., 1948. On the total reflection of plane waves. Quart. J. Mech. Appl. Math. 1, 376-384. 
Kaplunov, J., Zakharov, A., Prikazchikov, D.A., 2006. Explicit models for elastic and piezoelastic surface waves. IMA J. Appl. Math. 71, 768-782.

Kaplunov, J., Nolde E., Prikazchikov, D.A., 2010. A revisit to the moving load problem using an asymptotic model for the Rayleigh wave. Wave Motion 71, 440-451.

Kaplunov, J., Prikazchikov, D.A., Rogerson, G.A., 2013. On the edge bending wave in a thin elastic plate. Submitted for publication.

Kiselev, A.P., Parker, D.F., 2010. Omni-directional Rayleigh, Stoneley and Schölte waves with general time dependence, Proc. Roy. Soc. A. 466, 2241-2258.

Kiselev, A. P., Rogerson, G. A., 2009 Laterally dependent surface waves in an elastic medium with a general depth dependence. Wave Motion 46(8), 539-547.

D.F. Parker, Evanescent Schölte waves of arbitrary profile and direction, Europ. J. Appl. Math. 23 (2012) 267-287.

Royer, D., Dieulesaint, E., 1996. Elastic waves in solids II, Springer, Berlin.

T.C.T. Ting, (1996) Positive definiteness of anisotropic elastic constants, Mathematics and Mechanics of Solids, 1, pp. 301-314. 